\title{
Evidence-based postural assessment for use in therapy and rehabilitation
}

\section{JG Warren, J Bettany-Saltikov, P van Schaik, S Papastefanou}

Clinical assessment of spinal dysfunction involves the evaluation of back shape or symmetry. Variations in symmetry, shape or balance are generally believed (in conjunction with other symptoms) to be indicators or possible causes of dysfunction. With the onset of clinical governance, evidence-based practice has become paramount in all aspects of health. Numerous non-invasive objective methods varying in sophistication, cost and portability have been developed to measure and document back shape and posture.

The purpose of this current study was to assess the reliability of a new low-cost system on healthy young subjects. Intrarater reliability, using Pearson's correlation and intraclass correlation coefficients of landmark points on 50 'normal' subjects using a portable digital system, is reported. The results indicate that the system is reliable $(\mathrm{P}<0.001)$ and provides data suitable for evidence-based practice in clinical rehabilitation.

Key words: spinal dysfunction, evidence-based practice, clinical governance, postural assessment Warren JG, Bettany-Saltikov J, van Schaik P, Papastefanou S (2005) Evidence-based postural assessment for use in therapy and rehabilitation. Int J Ther Rehabil 12(12): 527-32

$\mathrm{S}$ pinal musculoskeletal dysfunction is a significant problem in Western society, with $70-85 \%$ of the general population experiencing one or more episodes of back problems during adult life and the annual prevalence ranging 15-45\% (National Institute of Health Guide, 1997). In the UK, disability resulting from back pain has an annual cost to the NHS of an estimated $£ 1.6$ billion pounds (Klaber-Moffat et al, 1999; Somani, 2001).

Physiotherapy plays a key role in the management of back disorders, treating in excess of 1.3 million people each year in Britain (Foster et al, 1999). Causes of back dysfunction include poor sedentary habits, lack of postural awareness, postural abnormalities, spinal deformities, degenerative and systemic diseases and neurological diseases, among others (Maggee, 2002; Pengel et al, 2003).

Clinical assessment of spinal dysfunction involves the evaluation of back shape or symmetry. Variations in symmetry, shape or balance are generally believed (in conjunction with other symptoms) to be indicators or possible causes of dysfunction (Maggee, 2002). Bullock-Saxton (1993) states that:

'While there is no certainty that the patients' posture is the cause of the signs or symptoms, the possibility of such a relationship needs to be acknowledged

\section{and postural changes monitored as} treatment progresses."

Traditionally, physiotherapists and other orthopaedic clinicians have relied on observation (unaided visual non-standardized assessment) in conjunction with brief subjective histories to diagnose back postural abnormalities and/or progression of postural deviations. This method of assessment is prone to error and is ostensibly subjective. Objective measurement of postural parameters may improve diagnostic accuracy, differentiation of spinal conditions and determination of patients' progress or deterioration (Bullock-Saxton, 1993; Maggee, 2002).

With the onset of clinical governance, evidencebased practice has become paramount in all aspects of health. Numerous non-invasive objective methods varying in sophistication, cost and portability have been developed to measure and document back shape and posture (Moreland et al, 1983; Bunnell, 1984; Houghton et al, 1986; Lovell et al, 1989; Singer et al, 1999). Existing low-tech measurement systems are generally low in cost and simple to use, but are limited in the measurements that can be made and generally can only document curves in one plane (Thulborne and Gillespie, 1976; Willner, 1981).

The contour body tracer (Thulborne and Gillespie, 1976), spinal pantograph (Willner, 1981) and flexirule (Lovell et al, 1989) are the most com-
JG Warren is Senior Lecturer, School of Computing, J BettanySaltikov is Senior Lecturer, School of Health and Social Care and $\mathbf{P}$ van Schaik is Reader, School of Social Sciences and Law, University of Teesside, Middlesbrough, Cleveland TS1 3BA, UK. S Papastefanou is Senior Spinal Consultant, Department of Orthopaedics, James Cook University Hospital, Middlesbrough, UK.

Correspondence to: JG Warren 
mon tactile methods reported. However, reports suggest that these methods are mainly used in research and rarely used in clinical practice (Kipling, 2001).

Hi-tech methods are costly because they are generally based on optical techniques requiring computer processing. Although highly accurate, they tend to be cumbersome to move and expensive to maintain. Moiré topography electronic measurement (Moreland et al, 1983; Tartaro and Austin, 1986), the Integrated Shape Imaging System (ISIS; Oxford Metrics Ltd, Oxford, UK) (Turner-Smith, 1988), rasterography (Hierholzer, 1999) and the Quantec System (Quantec Image Processing Ltd., Lancashire, UK) (Wojcik et al, 1994) are the most widely used systems. However, owing to their cost (more than $£ 30,000$, except for Moiré, which without electronics costs about £1000), requirement for experienced operator and complexity, the latter three systems are usually only accessible to specialist orthopaedic units, generally for the assessment of spinal deformities.

Further, slow uptake of these systems after initial excitement calls into question the clinical usefulness of the results as provided at the present time. Currently, objective measurement systems for postural evaluation are not widely available, especially in the NHS. Owing to financial constraints, many physiotherapy and orthopaedic clinicians who would benefit from such a system still have to rely on qualitative visual methods to assess posture and shape.

The Middlesbrough Integrated Digital Assessment System (MIDAS) is a new, low-cost (less than $£ 5,000$ ) portable and evidence-based system for the measurement of back shape and posture. The system, which has previously been described in a separate article (Warren et al, 2002), was found to be highly reliable (intrarater $r=0.99, P<0.0001$ ) on an anatomical mannequin. The purpose of this current study was to assess the reliability of the same system on healthy young subjects.

\section{SUBJECTS}

A convenience sample of 50 'healthy and asymptomatic' physiotherapy students was used in this study. Their ages ranged $19-41$ years (mean $=22.9$ years). There were 22 male and 28 female students with a mean body mass index (BMI) of 23 (ranging 16.9-30.3). Any subjects previously diagnosed with an associated orthopaedic problem were excluded from the study. Ethics approval was obtained from the local research ethics committee and informed consent was obtained prior to any measurements.

\section{INSTRUMENTATION}

The Microscribe 3DX Digitizer from Immersion Corp Ltd. (California) is a three-dimensional (3D) mechanical digitizer, developed for the computer graphics and engineering industries to create $3 \mathrm{D}$ computer models from physical clay, plastic or engineering parts. It is not dissimilar in its construction to a pantograph. It comprises an articulated, five-linkage, counterbalanced arm, which allows the stylus tip freedom of movement within a spherical volume of $0.63 \mathrm{~m}$ radius. Optical encoders in the joints record the joint rotations, allowing the measurement of joint angles from which the position of the stylus can be calculated (Figure 1).

The $\mathrm{x}, \mathrm{y}$ and $\mathrm{z}$ coordinates (Figure 2) derived give the $3 \mathrm{D}$ location of the stylus tip relative to its initial position. In Figure 2, the frontal, sagittal and horizontal planes correspond to the $\mathrm{xy}, \mathrm{yz}, \mathrm{zx}$ planes respectively. Mounting on a tripod enables it to be positioned correctly within its working range. Connected to a PC or laptop by a serial port, data can be collected and displayed from the digitizer.

\section{PROCEDURE}

Subjects were attired such that the whole of the back surface was visible. In total, 15 landmark points were palpated and marked with $8 \mathrm{~mm}$ diameter blue self-adhesive stickers on the subject's back as shown in Figure 3, while in a standing position. The person then stood on a wooden platform in a relaxed position with his/her feet either side of a central block (20 cm wide), thus gaining standardization of foot position throughout the sample.

Previous work (Bettany and Harrison, 1992a) has shown that standardization of foot position can significantly improve scan reliability. Points were recorded by depressing a foot pedal when the stylus

Figure 1. The Microscribe 3DX Digitizer in rest position.

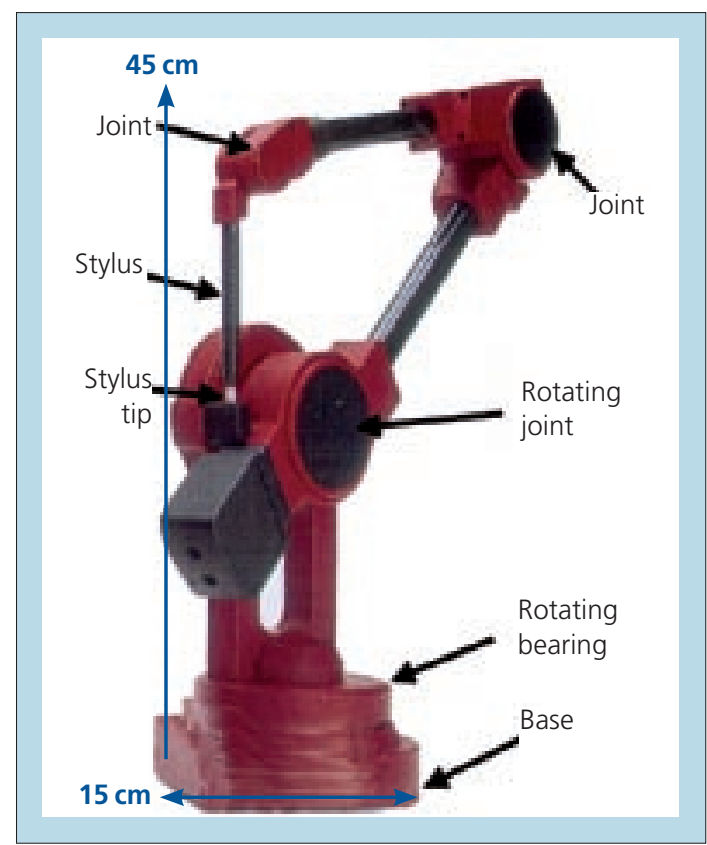




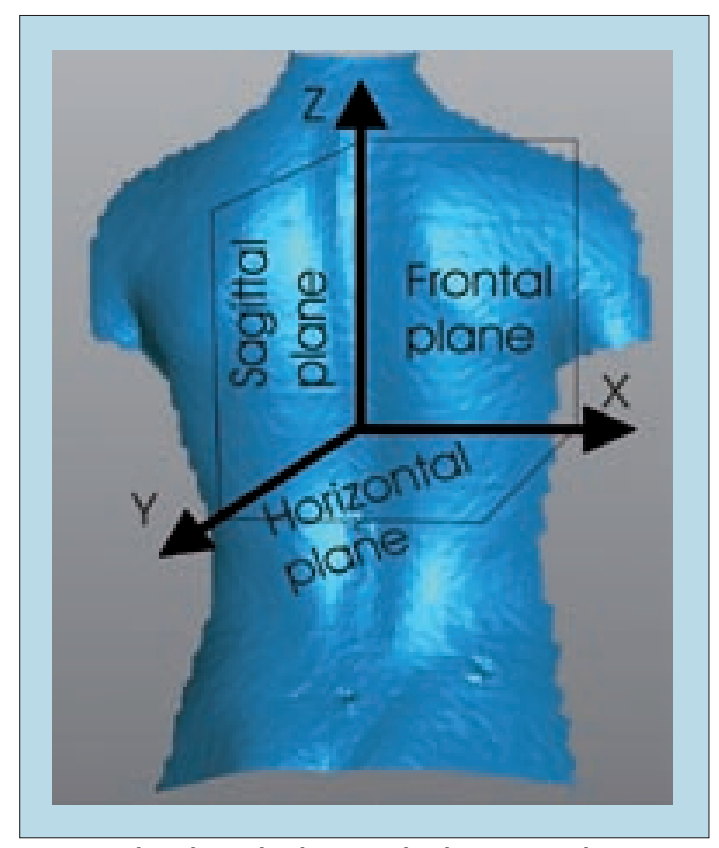

Figure 2. The relationship between the digitizer coordinate system and $\mathbf{x}$-ray planes.

tip was placed in contact with the marker. An audible signal was then produced and the coordinates were stored on computer disk. The subjects were allowed to relax for a minute and then recorded for a second time.

\section{SELECTION OF ANATOMICAL LANDMARKS}

The landmarks above were carefully chosen from current clinical methods, back shape studies and studies related to spinal deformities (Turner-Smith, 1988; Hierholzer, 1999; Leroux et al, 2000; O’Haire and Gibbons, 2000). The intention was to produce a contour tracing (map) for the cervical, thoracic and lumbar regions of the whole back (Figure 4, Table 1). The terminal vertebrae of the cervical, thoracic and lumbar curves were selected $(\mathrm{C} 2, \mathrm{VP}, \mathrm{T} 12, \mathrm{SA})$ as well as the apical vertebrae for each curve (CA, TA, LA).

Figure 3. Procedure for recording anatomical landmarks.

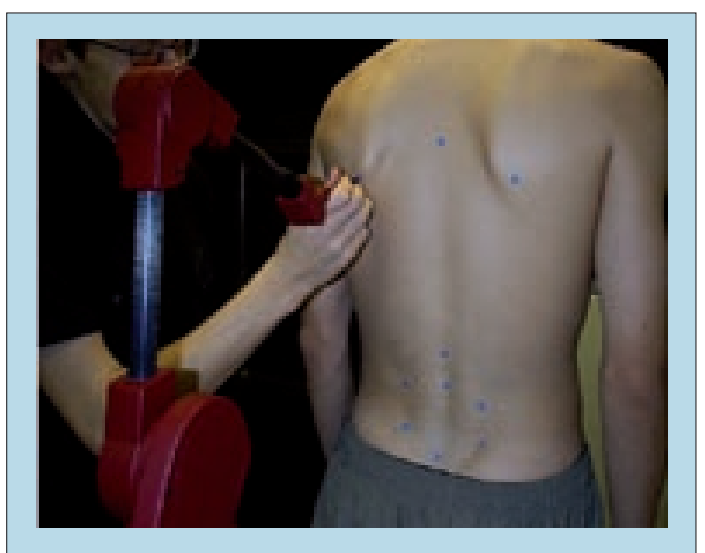

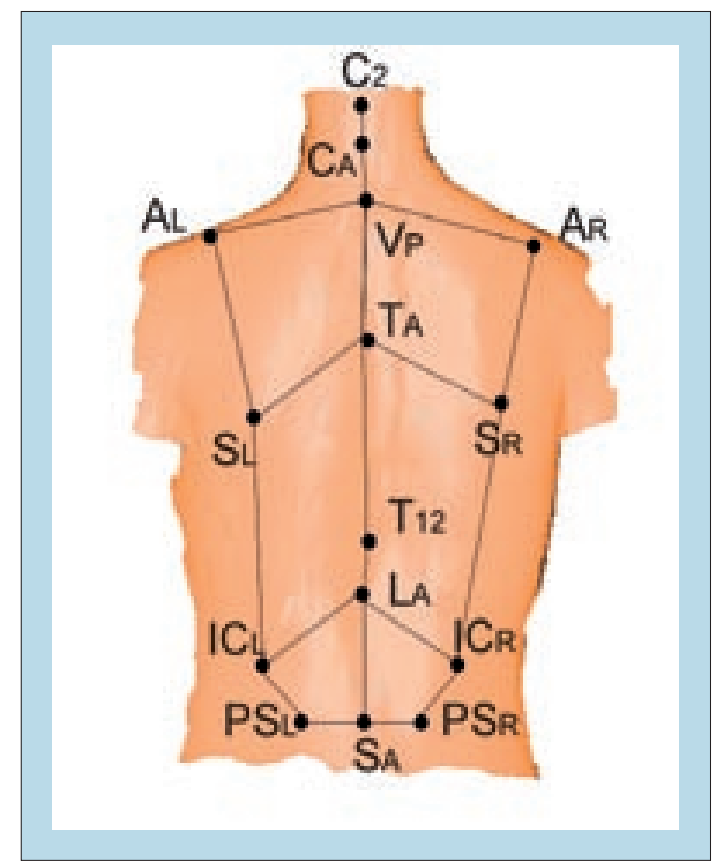

Figure 4. Definition of anatomical landmarks describing back shape and symmetry.

For landmarks on either side of the back, bony points were chosen that were as far from the spine as possible to enable a complete representation of back shape. A 'normal' back shape profile in three dimensions could then be produced that enabled the comparison of the two sides of the back, allowed asymmetries to be identified and described 'normal back shape'.

\section{DATA ANALYSIS}

Correlational analysis such as Pearson productmoment captures in a single index the association or relationship between two variables. It may be

\section{TABLE 1.}

Key to labelling of anatomical landmarks

\begin{tabular}{|c|c|}
\hline Label & Description \\
\hline $\mathrm{AL}$ & Left acromion process \\
\hline$A R$ & Right acromion process \\
\hline SL & Left inferior angle of scapula \\
\hline$S_{R}$ & Right inferior angle of scapula \\
\hline ICL & Left iliac crest \\
\hline$I C R$ & Right iliac crest \\
\hline PSL & Left posterior superior illiac spine \\
\hline PSR & Right posterior superior illiac spine \\
\hline $\mathrm{C}_{2}$ & 2nd cervical vertebrae \\
\hline CA & 4th cervical vertebrae \\
\hline$V_{p}$ & 7th cervical/1st thoracic \\
\hline TA & Apical thoracic vertebrae \\
\hline $\mathrm{T} 12$ & 12th thoracic vertebrae \\
\hline LA & Lumbar apical vertebrae \\
\hline SA & Sacrum point \\
\hline
\end{tabular}


helpful to think of correlation (or correspondence) in terms of interdependence of variables, so if one variable changes, the other one will too.

In this study, the association between repeated measures of $\mathrm{x}, \mathrm{y}$ and $\mathrm{z}$ coordinates for all points were analysed using Pearson's correlation coefficients (Batavia, 2001). Further, the intraclass correlation coefficient (ICC) was used to examine the agreement (or similarity) between pairs of scores, in addition to their correspondence. A significance level of 0.05 was used for statistical tests.

\section{RESULTS}

\section{Association or correspondence}

Pearson's correlation coefficient analysis on the 15 points in the xy, yz and zx planes demonstrated a

\begin{tabular}{l|llll}
$\begin{array}{l}\text { TABLE 2. } \\
\text { Pearson correlations (P>0.0001) }\end{array}$ & & & \\
Label & \multicolumn{1}{c}{ Description } & X axis & Y axis & Z axis \\
\hline AL & Left acromion process & $r=0.927$ & $r=0.943$ & $r=0.998$ \\
\hline AR & Right acromion process & $r=0.994$ & $r=0.941$ & $r=0.998$ \\
\hline SL & Left inferior angle of scapula & $r=0.993$ & $r=0.955$ & $r=0.998$ \\
\hline SR & Right inferior angle of scapula & $r=0.998$ & $r=0.945$ & $r=0.998$ \\
\hline ICL & Left iliac crest & $r=0.988$ & $r=0.947$ & $r=0.998$ \\
\hline ICR & Right iliac crest & $r=0.981$ & $r=0.939$ & $r=0.998$ \\
\hline PSL & Left posterior superior illiac spine & $r=0.985$ & $r=0.942$ & $r=0.998$ \\
\hline PSR & Right posterior superior illiac spine & $r=0.968$ & $r=0.931$ & $r=0.998$ \\
\hline C2 & 2nd cervical vertebrae & $r=0.957$ & $r=0.949$ & $r=0.998$ \\
\hline CA & 4th cervical vertebrae & $r=0.960$ & $r=0.937$ & $r=0.999$ \\
\hline VP & 7th cervical/1st thoracic & $r=0.940$ & $r=0.951$ & $r=0.999$ \\
\hline TA & Apical thoracic vertebrae & $r=0.956$ & $r=0.909$ & $r=0.998$ \\
\hline T12 & 12th thoracic vertebrae & $r=0.964$ & $r=0.955$ & $r=0.999$ \\
\hline LA & Lumbar apical vertebrae & $r=0.958$ & $r=0.952$ & $r=0.998$ \\
\hline SA & Sacrum point & $r=0.946$ & $r=0.954$ & $r=0.996$ \\
\hline
\end{tabular}

TABLE 3.

Intraclass correlation coefficients $(P>0.0001)$

\begin{tabular}{l|llll}
\hline \multicolumn{1}{c}{ Label } & \multicolumn{1}{c}{ Description } & X axis & Y axis & Z axis \\
\hline AL & Left acromion process & $r=0.9187$ & $r=0.9419$ & $r=0.9975$ \\
\hline AR & Right acromion process & $r=0.9945$ & $r=0.9400$ & $r=0.9975$ \\
\hline SL & Left inferior angle of scapula & $r=0.9928$ & $r=0.9488$ & $r=0.9978$ \\
\hline SR & Right inferior angle of scapula & $r=0.9883$ & $r=0.9428$ & $r=0.9980$ \\
\hline ICL & Left iliac crest & $r=0.9878$ & $r=0.9451$ & $r=0.9982$ \\
\hline ICR & Right iliac crest & $r=0.9802$ & $r=0.9383$ & $r=0.9972$ \\
\hline PSL & Left posterior superior illiac spine & $r=0.9856$ & $r=0.9403$ & $r=0.9981$ \\
\hline PSR & Right posterior superior illiac spine & $r=0.9642$ & $r=0.9308$ & $r=0.9982$ \\
\hline C2 & 2nd cervical vertebrae & $r=0.9537$ & $r=0.9438$ & $r=0.9981$ \\
\hline CA & 4th cervical vertebrae & $r=0.9578$ & $r=0.9314$ & $r=0.9987$ \\
\hline VP & Vertebra prominens & $r=0.9599$ & $r=0.9463$ & $r=0.9992$ \\
\hline TA & Apical thoracic vertebrae & $r=0.9547$ & $r=0.9466$ & $r=0.9984$ \\
\hline T12 & 12th thoracic vertebrae & $r=0.9626$ & $r=0.9479$ & $r=0.9984$ \\
\hline LA & Lumbar apical vertebrae & $r=0.9571$ & $r=0.9479$ & $r=0.9981$ \\
\hline SA & Sacrum point & $r=0.9446$ & $r=0.9515$ & $r=0.9962$ \\
\hline
\end{tabular}

correlation coefficient value of between $r=0.92$ and $r=0.99(P<0.0001)$ for all points in all three planes (Table 2).

\section{Agreement}

ICC analysis on the 15 points in the $x y, y z$ and zx planes demonstrated an ICC value of between $r=0.9187$ and $r=0.9984(P<0.00001)$ for all points in all three planes (Table 3$)$.

\section{DISCUSSION}

Previous work by Warren et al (2002) on a mannequin demonstrated that the inherent accuracy of a contact mechanical system was extremely high $(r=0.999, P<0.0001)$ when using pre-palpated points. The current work confirms that the digitizer is also extremely accurate and reliable in the measurement of back standing posture in a sample of 'normal' subjects with a mean BMI of 23.

Intrarater correspondence as well as agreement measures were statistically significant $(P<0.001)$ for all points in the frontal, horizontal and sagittal axes (xy, yz and zx planes). The accuracy of results are comparable to those reported from other high-tech systems (Turner Smith, 1988; Bettany et al, 1992b; Burwell et al, 1983; Wojcik et al, 1994; Hierholzer, 1999). Unfortunately, direct comparisons between these systems were not possible as different measures were used as a basis for calculating reliability.

Reliability and reproducibility are prerequisites for monitoring changes in back posture and efficacy of therapeutic interventions. The onset of evidence-based practice and clinical governance necessitates objective documentation of patient records to demonstrate effective assessment, treatment and management of patient care. However, further studies on different patient groups are required to provide further evidence of validity and sensitivity. The authors are hoping to complete this study in a more general population.

In addition, the uptake of any system in clinical practice requires a high level of acceptance among clinicians. This has been addressed in a previous study by van Schaik et al (2002), who studied the clinical acceptance of the digitizer for postural assessment as perceived by physiotherapists. Clinicians showed high levels of technology acceptance in terms of the three components perceived ease of use, perceived usefulness and intention to use.

The system should be particularly well suited to use in small clinical units, owing to its simplicity of operation, size and cost. The existing software allows calculation of height differences relative to fixed points on the spine. Future studies conducted by the authors will attempt to determine clinically relevant 'normative' values from the measured points. The intention will be to produce a topographical map of 
standing back posture. This work is necessary for implementation of the system in clinical practice.

Known limitations, which the authors intend to quantify, are marking inconsistencies between different markers owing to palpation and the time gap between successive measurements. However, the former was not a cause of inconsistencies in the current study because marking was done only once for each subject by the same marker. In order to fully test consistency, the marking would have to be done by different markers and the measurements separated by a week or more.

The landmark points shown in Figure 4 form the basis for measurements of the back shape and hence the underlying spinal deformity. Since they are measured in three dimensions, they show not only the asymmetry and curvature in the frontal plane, but also the corresponding changes in the saggital plane and the horizontal.

\section{CONCLUSIONS}

Initial results indicate the system to be a very reliable and accurate method of recording the 3D position of landmark points on the back and a promising technique for capturing back shape. The proposed system is portable and of low cost, and initial results suggests that it may be useful in a number of clinical settings - for example, physiotherapy and orthopaedic medicine, as well as spinal units.

Use of this system as an adjunct to traditional methods may also increase the effectiveness of postural management (Farley et al, 2000). The next phase of the research is to assess the system's interrater reliability on healthy asymptomatics and patients with postural dysfunction and spinal deformities. Further research will investigate the presence of different back profiles and possible spinal indicators for back dysfunction. Spinal deformity requires the measurement of more points on the spine. Further research is required to establish the effect of different persons palpating and marking on outcomes. IJTR

Conflict of interest: none.

Batavia M (2001) Clinical Research for Health Professionals A User Friendly Guide. Butterworth-Heinemann, Boston

Bettany JA, Harrison DJ (1992a) The Stanmore footplate: an improved method in ISIS optical scanning. In: Alberti A, Drerup B, Hierholzer E, eds. Surface Topography and Spinal Deformity. Gustav Fischer Verlag, Stuttgart: 42-6

Bettany JA, Harrison DJ (1992b) The ISIS experience at the Royal National Orthopaedic Hospital. In: Alberti A, Drerup B, Hierholzer E, eds. Surface Topography and Spinal Deformity. Gustav Fischer Verlag, Stuttgart: 70-5

Bullock-Saxton J (1993) Postural alignment in standing: a repeatability study. Aust Physiother 39(1): 25-9

Bunnell WP (1984) An objective criterion for Scoliosis Screening. J Bone Joint Surg 66A: 1381-7

Burwell RG, James NJ, Johnson F, Webb JK, Wilson G (1983) Standardised trunk asymmetry scores. A study of back contour in healthy school children. J Bone Joint Surg 65B(4): 452-63

Farley R, Clark J, Davidson C et al (2000) What is the evidence for the effectiveness of postural management? Int $J$ Ther Rehabil 10(10): 449-55
Foster NE, Thompson KA, Baxter GD, Allen JM (1999) Management of nonspecific low back pain by physiotherapists in Britain and Ireland. Spine 24(13):1332-42

Hierholzer E (1999) Valuation of body surface measurements: accuracy rating of anatomical landmarks. In: Stokes IAF, ed. Research into Spinal Deformities 2. Studies in Health Technology and Informatics. IOS Press, Amsterdam: 25-8

Houghton GR, Harris JD, Jefferson RJ, Turner-Smith AR (1986) Measurement of back shape using ISIS scanning. In: Harris JD, Turner-Smith AR, eds. Surface Topography and Spinal Deformity. Gustav-Fischer Verlag, Stuttgart: 197-204

Kipling K (2001) Assessment of posture: just eyeballing it? Unpublished thesis, University of Teesside, Middlesbrough

Klaber-Moffat J, Torgerson D, Bell-Syer S et al (1999) Randomised controlled trial of exercise for low back pain: clinical outcomes, costs and preferences. Br Med J 319: 279-83

Leroux MA, Zabjek K, Simard G, Badeaux J, Coillard C, Rivard $\mathrm{CH}$ (2000) A noninvasive anthropometric technique for measuring kyphosis and lordosis. Spine 25(13): 1689-94

Lovell FW, Rothstein JM, Personius WJ (1989) Reliability of clinical measurements of lumbar lordosis taken with a flexible rule. Phys Ther 69(2): 342-5

Maggee DJ (2002) Orthopaedic Clinical Assessment. 4th edn. Saunders, Philidelphia: 697-726

Moreland MS, Pope MH, Stokes IAF, Weierman R (1983) The clinical use of Moire topography for spinal deformity. A perspective challenge. In: Drerup B, Frobin W, Hierholzer H, eds. Moire Fringe Topography and Spinal Deformity. GustavFischer Stuttgart, New York: 129-40

National Institute of Health Guide (1997) Research of low back pain and common spinal disorders. http://grants.nih.gov/grants guide/pa-files/PA-97-058.html (accessed 8/12/2003)

O'Haire C, Gibbons P (2000) Inter-examiner and intra-examiner agreement for assessing sacroiliac anatomical landmarks using palpation and observation: pilot study. Man Ther 5(1): 13-20

Pengel LH, Herbert RD, Maher CG, Refshauge KM (2003) Acute low back pain: systematic review of its prognosis. $\mathrm{Br}$ Med $J$ 327: $327-3$

Singer KP, Goh S, Leedman P, Price RI (1999) Reliability of thoracic kyphosis. In: Stokes IAF, ed. Research into Spinal Deformities 2. Studies in Health Technology and Informatics. IOS Press, Amsterdam: 29-32

Somani K (2001) Activity and pain killers best for back pain. $\mathrm{Br}$ Med J 322: 318

Thulborne T, Gillespie R (1976) The rib hump in idiopathic scoliosis. Measurement analysis and response to treatment. $J$ Bone Joint Surg 58B: 456-62

Tartaro M, Austin JH (1986) Moire topography in scoliosis. Accuracy of assessing lateral curvature as a function of the region of the curve. In: Harris JD, Turner-Smith AR, eds. Surface Topography and Spinal Deformity. Gustav-Fischer Verlag, Stuttgart: 125-33

Turner-Smith AR (1988) A television/computer three dimensional surface shape measurement system. J Biomech 21(6): 515-29

van Schaik P, Bettany-Saltikov JA, Warren JG (2002) Clinical acceptance of a low-cost portable system for postural assessment. Behav Inf Technol 21(1): 47-57

Warren JG, Bettany-Saltikov JA, Van-Schaik P (2002) 3-D measurement of posture and back shape using a low cost, portable system a reliability study. In: Tanguy A, Peuchot B, eds. Research into Spinal Deformities. IOS Press, Amsterdam: 100-4

Willner S (1981) Spinal pantograph-A non-invasive technique for describing kyphosis and lordosis in the thoraco-lumbar spine. Acta Orthop Scand 52: 525-9

Wojcik AS, Phillips GF, Mehta MH (1994) Recording of the back surface and spinal shape by Quantec image system: a new technique in the scoliosis clinic. J Bone Joint Surg 76B(suppl.): 15

\section{KEY POINTS}

- The Middlesbrough Integrated Digital Assessment System has a very high intrarater reliability $(P<0.001)$

- The system has relatively low cost and high portability compared to other high-tech systems.

It has a high clinical acceptance in terms of ease of use, usefulness and intention to use.

It can be applied to a range of clinical areas. 
There has been a plethora of studies describing methodologies for back surface measurement (Alberti et al, 1992). Methods for optical measurement that have been developed and reported include stereophotography, Moiré topography and rasterstereography. Optical scanning methods are also used, which provide spatial information relating to the 3D shape of the back.

While most non-contact systems are expensive, this study describes a simple, inexpensive method for spine and back assessment. The reported investigation is a continuation of a previous study from the same authors (Warren et al, 2002), which used a tactile 3D digitizer on a mannequin. This digitizer demonstrated high measurement accuracy when using predetermined anatomical points.
The results reported within this article also indicate that the digitizer system is reliable and accurate in measuring the 3D coordinates of various landmarks in normal subjects during quiet standing.

While the authors indicate that the results are comparable to previous results reported using other systems, they also point out that further studies on clinical populations are required. To the advantage of the reader, this study makes use of anatomical landmarks that have been previously validated by other reports.

Although the results presented in this article are encouraging, the authors should continue their work to validate their methodology and the system on patients with musculoskeletal conditions. If this system can be further developed and validated for functional measurement, it will help in assessing the range of movement of the spine.

While using anatomical landmarks for clinical application and measurement, it is important that the relationship between various points remain constant with variations in posture. Although there is still a paucity of information to derive such relationship, if this can be established, the amount of ionizing radiations that are currently used could greatly be reduced. The system reported within this study has the potential to fill this gap and this would lead to new inexpensive avenues in the functional evaluation of the back.

In this context, further studies on the effects of day-to-day repeatability and errors owing to placing markers on the landmarks need to be carried out. This should lead to the assessment of errors introduced by the skin movement in dynamic measurement.

Although the difficulties for novice operators who are unfamiliar with palpating and locating the spinous processes should be considered, this difficulty could be overcome by limiting the number of markers placed on the subject. This could be the natural progression for this study.

The reported technique promises to be an inexpensive methodology for back assessment, which has a potential for further development.

\section{Nachiappan Chockalingam}

Reader in Biomechanics

Faculty of Health and Sciences

Staffordshire University

Stoke on Trent ST4 2DF UK

Alberti A, Drerup B, Hierholzer E (1992) Surface Topography and Spinal Deformity. Gustav Fischer Verlag, Germany
Surface topography and back surface measurement has a long research history. The ideas embodied in the various reports of the Research Society for Spinal Deformities cover a range of systems, ranging from Moiré topography to laser and coherent light scanning.

These techniques all have their strengths and weaknesses. The major problems are those of relatively high cost, since the equipment generally demands computer- and camera-based equipment in dedicated space with a trained operator, and repeatability accuracy. However, the development of better digital cameras and lower-cost computers have undoubtedly driven this cost downward in the last few years.

In the context of health-care delivery the problems may be viewed as a disadvantage, since there is often no clear cost benefit offered by the new technologies to patient management and treatment over conventional methods of clinical observation and radiology.

In the field of spinal deformity conditions, themselves relatively rare in the general population, the vari- ous systems described over the years have tended to remain a research tool rather than a practical diagnostic and management instrument.

The advent of magnetic resonance imaging and real-time and $3 \mathrm{D}$ ultrasound is now frequently a routine part of clinical investigation, changing the way spinal problems are regarded by surgeons and others and often obviating the need for surface measurement. This is partly because the true relationships between the surface deformity and underlying structural changes of anatomy are still unclear

This article is a further development of a technique described in an earlier communication by the same group. The previous report was on a laboratory-based 3D analysis system, using appropriate dummy material, whereas the present study describes the application of this technique to normal subjects.

This is an important stage in the development of this work, since application of these techniques to biological and clinical environments often reveals problems missed in the theoretical situation. By establishing accuracy, the reader can make a judgment on the application of the equipment.

Anatomical landmarks are recognized to be difficult to accurately identify, owing to skin movements or obesity, difficulty in location by palpation and issues of subject cooperation, in the case of a child. The authors note the problems in their article and have overcome some of these by using one operator to undertake the location of landmarks.

However, for others to apply the same technique using the digitizer, it is important to know the interand intraobserver error status for each anatomical point and how much potential error can arise form the issues of skin movement and so on. Further, anatomical variation occurs in normal individuals and this is undoubtedly magnified in cases such as scoliosis, suggesting that a considerable amount of more work is required to validate these points.

Another problem affecting all surface topography techniques is the repeatability of measurements from visit to visit. Children and adults with skeletal and other deformities often show progression and these changing parameters, as well as issues such as respiratory movements, need addressing.

Foot placement is vitally important, but this alone cannot address the bigger problems. There still remains the problem of the relationship between the surface 'map' of the trunk and the underlying skeletal and soft tissue anatomy and the authors will need to consider this in their future work.

The group has developed an inexpensive and potentially useful tool for application in the spinal clinic. It needs further technique validation in the context of different users and their accuracy in locating, and repeated identification, of the same landmarks. If the group can overcome these problems of anatomy, technique and patient dynamics, as well as the application to the spinal deformity case, then its system may gain the all-important clinical uptake for routine patient evaluation.

\section{Peter H Dangerfield}

Director, Phase 1

School of Medical Education

University of Liverpool

Liverpool L69 3GE

UK 